\begin{tabular}{|c|c|c|c|c|}
\hline 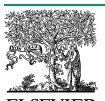 & Review & TRENDS in Endocrinology and Metabolism & Vol.15 No.2 March 2004 & $\begin{array}{l}\text { Full text provided by www.sciencedirect.com } \\
\text { science DiRECT. }\end{array}$ \\
\hline
\end{tabular}

\title{
Estrogen, inflammation and cardiovascular risk in women: a critical appraisal
}

\author{
Stefan Störk ${ }^{1,2}$, Yvonne T. van der Schouw ${ }^{2}$, Diederick E. Grobbee ${ }^{2}$ and Michiel L. Bots ${ }^{2}$ \\ ${ }^{1}$ Cardiology, Julius-Maximilians University Würzburg, Würzburg, Germany \\ ${ }^{2}$ Julius Center for Health Sciences and Primary Care, University Medical Center Utrecht, Utrecht, The Netherlands
}

\begin{abstract}
The widely shared enthusiasm about the cardioprotective potential of estrogenic compounds has come to an abrupt halt since randomized trials failed to show a cardiovascular risk reduction in postmenopausal women. This was unexpected because observational studies had strongly suggested that hormone replacement therapy would reduce the incidence of cardiovascular disease. Inflammatory activity is considered central in atherogenesis and atherosclerosis progression. Thus, parts of the striking discrepancy between observational and randomized data have been attributed to an estrogen-mediated adverse effect on inflammation. Here, we review the current clinical evidence with respect to the inflammation-modulating effects of different estrogenic compounds as one potential explanatory factor for these divergent findings. We conclude that it is still unclear whether estrogen-modulated inflammation is an important biological factor determining clinical outcome or a mere epiphenomenon.
\end{abstract}

The universally higher coronary heart disease (CHD) risk for men and the increased CHD risk for women experiencing early menopause [1] led to the hypothesis that endogenous estrogen and exogenous sex hormones [opposed (HRT) and unopposed (ERT) hormone replacement therapy] might be cardioprotective in women [2] Indeed, observational data had suggested a $40-50 \%$ cardiovascular risk reduction in postmenopausal women using HRT [3-5]. To arrive at definitive conclusions, controlled clinical trials on the effects of ERT/HRT in primary [6-8] and secondary prevention settings [9-18] were initiated. To date, 13 trials reported on clinical events in coronary artery $[8-10,15,16]$, cerebrovascular $[8,13,14]$ and peripheral artery disease [12], or surrogate markers of atherosclerosis as endpoints $[6,7,11,17,18]$. All but one of these trials [7] yielded neutral or negative results. In addition, several trials reported an increased risk for thromboembolic disease, such as deep venous thrombosis and pulmonary embolism $[8,19]$.

In primary prevention, the Women's Health Initiative studied 16608 women randomized to combined HRT with conjugated equine estrogens and medroxyprogesterone acetate or placebo. After 5.2 years of follow up, the data

Corresponding author: S. Störk (stoerk_s@klinik.uni-wuerzburg.de). caused the safety monitoring board to recommend terminating the trial because the overall risks exceeded the benefits: all cardiovascular outcomes and breast cancer risk showed increased incidence rates opposed to a beneficial effect in preventing bone fracture and a trend towards a reduced colon cancer rate [8]. Thus, the HRT regimens used in these trials did not offer cardioprotection (or might even be detrimental) in women who had passed menopause for $\sim 10-15$ years (the average age of women studied in randomized trials was $60-65$ years). Consequently, the guidelines on counseling postmenopausal women have been adjusted recently [20], and a conservative approach to initiation and long-term application of HRT has been adopted.

This review summarizes the current clinical evidence with respect to the inflammation-modulating effects of different estrogenic compounds as one potential explanatory factor for the divergent findings outlined above. The value of inflammatory markers as research tools is put into perspective and an outline of future research activities is proposed.

How can the divergent data be explained?

The striking discrepancy between observational and randomized data is apparent only with regard to the cardiovascular benefit or harm, whereas findings are consistent with regard to thromboembolic disease risk, stroke risk, bone protection, breast and colorectal cancer risk [21]. To explain this phenomenon, methodological issues [21] have been considered, including problems in patient selection (selection bias, healthy user bias), data collection (compliance bias, information bias) and data analysis (inadequate consideration of change in estrogen exposure over time, incomplete capture of early clinical events). Biological issues have also been explored, including differences among various hormone regimens and study populations, and adverse modulation of hemostasis, lipid subfractions, genetic susceptibility and inflammation. In particular, a proinflammatory effect of estrogenic compounds has been proposed because C-reactive protein (CRP) was found to increase following HRT. Furthermore, experimental studies delineated mechanisms through which estrogen might have proinflammatory effects by increasing tumor necrosis factor $\alpha$ (TNF- $\alpha)$-induced endothelial adhesion molecules [22] and by promoting an angiogenic phenotype [23]. 
Hence, the hypothesis of the 'attrition of the susceptible' was formulated: it assumes a cohort of women who are uniquely susceptible to estrogen-triggered side effects (possibly a combination of both prothrombotic and proinflammatory effects), which lead to early clinical events in randomized trials [24]. Currently, it is unclear to what extent each of these methodological and biological issues contributed to the selectively divergent findings from clinical trials of HRT and observational studies with respect to cardiovascular disease [24].

\section{Inflammatory processes are intertwined with atherosclerotic disease progression}

Vascular inflammatory processes are considered central in atherogenesis and are known to propagate each stage of atherosclerosis progression [25,26]. The major cardiovascular risk factors (cigarette smoking, hypertension, hyperglycemia and atherogenic lipoproteins) trigger noxious stimuli. These, in turn, elicit expression of cellular adhesion molecules, facilitating the attachment of monocytes to endothelial cells, and chemotactic factors, which promote migration of monocytes into the subendothelial space. Chemokines, such as monocyte chemoattractant protein 1 (MCP-1), augment the accumulation of macrophages, mast cells and activated T cells within the atherosclerotic lesion. Activated macrophages and upregulation of matrix metalloproteinases (MMPs) might be involved in the weakening of the fibrous cap of the plaque, rendering it prone to rupture. Every step in atherogenesis involves cytokines or other bioactive molecules, which promote inflammatory activity $[25,26]$.

Recently, much attention in the assessment of vascular inflammation has been given to CRP, which is one of the principal products of the acute-phase response, and which might also have a direct effect on its own amplification, regardless of the reason for this elevation [26-32]. CRP is largely, but not exclusively, regulated by interleukin- 6 (IL-6)-mediated biosynthesis [33]. IL-6 probably induces increased expression of the gene encoding CRP in the liver. Cytokines such as TNF- $\alpha$ can, in turn, stimulate the expression of IL-6 and leukocyte adhesion molecules [25,26].

However, the sources of circulating cytokines and inflammatory mediators remain unclear. They might arise from atheroma, reflecting the extent of inflammatory activity within the lesion, or nonvascular sources, reflecting inflammatory states, such as chronic infections [34]. Both vascular and nonvascular sources might prove important to various degrees in different individuals.

\section{Inflammatory markers as research tools in the assessment of inflammatory activity}

Serum/plasma markers of inflammation could be used to assess the degree of inflammation and provide insights into the pathophysiology of atherosclerosis [25,26,34,35]. Among the most widely accepted markers are proinflammatory risk indicators, such as oxidized low-density lipoproteins (LDLs); proinflammatory cytokines, such as IL-1 and TNF- $\alpha$; cell adhesion molecules, such as intercellular adhesion molecule 1 (ICAM-1), vascular cell adhesion molecule 1 (VCAM-1) and selectins; inflammatory stimuli with hepatic effects, such as IL-6; and products of hepatic stimulation, such as CRP and serum amyloid A (SAA) [35]. However, these markers are not only nonspecific for atherosclerosis-mediated inflammation, but are also raised in conditions of systemic inflammation (e.g. connective tissue diseases) or local infections (e.g. gingivitis, bronchitis or urinary tract infections). It is not known to what degree systemic levels of any inflammation marker directly reflect inflammation at the atherosclerotic lesion, but systemic levels of cell adhesion molecules were found to be closely related to levels measured in the coronary circulation [36].

\section{HRT modulates inflammatory activity}

Estrogens regulate and modulate important biological functions of different cellular components of the vascular wall, including the endothelium, smooth muscle cells, vascular stromal cells and different leukocyte species [37-39]. The involved signaling cascades are also integral parts of the inflammatory response to injury. Therefore, estrogen probably also acts as a major modulator of the inflammatory response $[25,40]$.

Numerous clinically controlled studies (most of them of short duration, i.e. weeks to a few months, and with relatively small numbers, i.e. fewer than 50 participants per group) have documented that estrogenic compounds modulate the inflammatory response. The markers studied comprise a large spectrum of specific and nonspecific inflammation markers, markers reflecting endothelial function and markers linking inflammation and coagulation. These studies are summarized in Table 1, ordered by investigated parameter. More detailed information with regard to study design, numbers per group and hormone preparations used is given in Tables $2 \mathrm{~A}$ and $2 \mathrm{~B}$ (see supplementary information Table $2 \mathrm{~A}$ and $2 \mathrm{~B}$, http://archive.bmn.com/supp/tem/Störk_Table2.pdf) for inflammatory and vasoactive mediators separately. Recently, it was shown that MCP-1, a particularly important mediator and amplifier of atherogenic signals, was downregulated after introduction and upregulated after discontinuation of $17 \beta$-estradiol; this observation was reproduced by others with conjugated equine estrogens $[41,42]$. Downregulation of inflammatory and vasoactive markers by different types, dosages and routes of HRT application has been demonstrated for cell adhesion molecules - such as ICAM-1, VCAM-1 and E-selectin [43-54] - TNF- $\alpha$ [55-57], vascular endothelial growth factor [58] and endothelin-1 [59-67]. As an endotheliumderived relaxing factor with anti-atherosclerotic and antiinflammatory properties, nitric oxide (NO) has received particular attention. It has been shown that estrogen participates in the transcriptional regulation of endothelial NO synthase and induces NO release through nongenomic mechanisms [68]. However, this upregulation at the cellular level has not unequivocally been reproduced in clinical studies $[61,63,69]$. Moreover, effects on other vasoactive markers, such as prostacyclin and thromboxane $\mathrm{A}_{2}$, yielded neutral results $[63,65]$.

A disturbing feature in many reports is the divergent regulation of markers that were originally thought to reflect similar inflammatory pathways. This might result mainly from the fact that the effects of estrogen on cell 
Table 1. Main findings of clinical studies on the effect of ERT/HRT on inflammatory and vasoactive mediators ${ }^{a, b}$

\begin{tabular}{|c|c|c|c|}
\hline Inflammatory mediator & Increased (Refs) & Unchanged (Refs) & Decreased (Refs) \\
\hline ICAM-1 & & [48] & {$[43,46,48,49,51,52,56,71,75,98]$} \\
\hline VCAM-1 & & [56] & {$[43,46,49,51,52,53,75,98]$} \\
\hline E-selectin & & & {$[43,46,47,49,51,52,56,64,72,98]$} \\
\hline CRP & {$[28,45,54,55,72-75,77,79,80]$} & {$[43,45,51,53,54,56,64,80]$} & {$[78,79,83]$} \\
\hline IL-6 & & {$[49,51,54]$} & \\
\hline TNF- $\alpha$ & & & [56] \\
\hline MCP-1 & & & {$[42,44,56]$} \\
\hline Fibrinogen & [72] & {$[56,74]$} & {$[43,45,64,73]$} \\
\hline \multicolumn{4}{|l|}{ Vasoactive mediator } \\
\hline ET-1 & & {$[63,65,66]$} & {$[59,60,63,64,67,69]$} \\
\hline NO & {$[61,69]$} & {$[63]$} & \\
\hline
\end{tabular}

${ }^{a}$ Abbreviations: CRP, C-reactive protein; ERT/HRT, unopposed/opposed hormone replacement therapy; ET-1, endothelin-1; ICAM-1, intercellular adhesion molecule 1; IL-6, interleukin-6; TNF- $\alpha$, tumor necrosis factor $\alpha$; MCP-1, monocyte chemoattractant protein 1; NO, nitric oxide; VCAM-1, vascular cell adhesion molecule 1.

${ }^{b}$ Studies with a randomized design are in bold. Appearance of a reference in different columns may be a consequence of various hormone regimens used or divergent results in important subgroups. For details refer to Tables 2A and 2B (see supplementary information Tables 2A and 2B, http://archive.bmn.com/supp/tem/Störk_Table2.pdf).

biology are complex. Estrogen controls transcription of a large variety of genes but also influences multiple signal transduction pathways through nongenomic mechanisms, which might lead to opposite biological effects depending on the triggering stimulus (for a review see [70]). For example, whereas cell adhesion molecules were lowered in most reports, CRP and MMP [51] were upregulated. Interestingly, this was only found consistently with conjugated equine estrogens $[8,28,32,45,55,71-75]$. The effect appeared to be strongest with unopposed conjugated equine estrogen [75] and was possibly ameliorated by the addition of a gestagen [76]. Conversely, six randomized trials with $17 \beta$-estradiol (three with oral $[43,64,77]$, two with transdermal administration [78,79], and one comparing both [80]) and four non-randomized studies [53,81-83] found reduced or unaffected CRP levels with either transdermal or oral combined regimens. Apparently, unopposed $17 \beta$-estradiol also raised CRP in a dose-dependent manner $[77,80,82]$. Thus, the importance of the first pass effect of oral unopposed estrogen, the ameliorating effect of gestagens on hepatic protein synthesis and the relevance of the estrogen-induced rise in CRP remain to be clarified. Estrogen-generated CRP could activate the complement cascade, thereby aggravating the thromboembolic risk, or could be unrelated to vascular inflammation, merely reflecting hepatic estrogen metabolism. Interestingly, IL-6 levels were not substantially affected by conjugated equine estrogens, as reported by three smaller randomized studies $[49,51,54]$. This implies that HRT-associated elevation of CRP is mediated by IL-6-independent pathways. These findings were corroborated and expanded by data from a nested case-control study of the Women's Health Initiative [32]. In this study, long-term HRT was associated with increased CRP, but HRT users appeared to be at similar risk compared with nonusers for any level of baseline CRP. The authors concluded that the baseline CRP level, rather than HRT use, was the primary determinant of risk in this study.

Inflammatory markers predict morbidity and mortality in postmenopausal women

Numerous prospective studies have shown a positive association between CRP and future cardiovascular events in various populations in primary and secondary prevention settings (reviewed in [84]). The available data imply a gradual increase in cardiovascular risk with increasing levels of CRP $[27,32,85,86]$ and also IL-6 $[32,85]$ in healthy, middle-aged and elderly women (about two to threefold increased risk for comparison between highest and lowest tertile of levels of respective marker). In general, a similar picture exists for SAA, ICAM-1 and P-selectin [30,87].

\section{Lowering of inflammatory markers might decrease cardiovascular risk}

Data from the Cholesterol and Recurrent Events trial indicated that long-term statin therapy lowered plasma levels of CRP, and the efficacy in lowering the event rate was greatest in those with increased levels of CRP [88]. This effect was also seen in other studies and might be independent of LDL cholesterol [89,90]. Several other preventive strategies to lower inflammation and possibly modify cardiovascular risk have been described, such as exercise, blood pressure lowering, weight reduction, smoking cessation, moderate alcohol consumption and improvement of insulin resistance. However, no study specifically addressed whether any of these interventions would also improve the clinical outcome via modulation of inflammation.

\section{...but ERT/HRT trials in postmenopausal women showing such effects on clinical endpoints are lacking}

Similarly, no endpoint studies reported on a hypothesized cause-and-effect relationship between lowered levels of inflammation markers following HRT and improved clinical outcome, such as cardiovascular event rate, or surrogate markers of atherosclerosis, such as intimamedia thickness. However, such trials were needed to determine to what extent inflammatory processes affect the cardiovascular risk associated with HRT use.

Alternatively, available databases of already published trials could be used. For example, it would be interesting to look at inflammatory markers in the only randomized clinical trial that reported intima-media thickness regression in postmenopausal women treated with unopposed 17ß-estradiol [7]. Apparently, this effect was limited to women not on lipid-lowering therapy (i.e. estrogen 
treatment only). Measurement of CRP and IL-6 levels would enable the testing of the post hoc hypothesis that estrogen induced regression by modulating inflammatory pathways. Similarly, the pro- and anti-inflammatory activity could be related to progression and regression using data from a randomized trial with tibolone, a synthetic estrogenic compound that also exerts androgenic and progestagenic actions, in which carotid intima-media thickness was assessed by ultrasound in 850 women in Europe and the USA over three years [91]. This study has been unblinded and results will be available soon.

\section{What is still missing in the HRT puzzle?}

Admittedly, the randomized world has its imperfections [92], but the evidence accumulated in the past 24 months has made it very clear that no overall benefit in chronic disease can be expected by long-term administration of combined HRT in women who have passed menopause by 10-15 years. In these women, detrimental effects outweighed benefits that might be realized via reduced bone fracture and colon cancer rate. The results of the trial arm of the Women's Health Initiative, which utilized unopposed estrogen, will provide important insights in a primary prevention strategy reserved for women after hysterectomy. It will enable a direct quantification of the impact of the addition of gestagen on inflammatory markers. Regarding the association with breast cancer risk, very recent results from the Million Women Study also support the concept of unopposed estrogen as the least detrimental option [93].

Because estrogenic compounds still have no rival with regard to the alleviation of perimenopausal symptoms, many women will continue to consider short-term hormone use for symptom relief. Future research should therefore be directed towards defining both mechanisms and markers of risk and benefit, in addition to describing subgroups of peri- and postmenopausal women who will experience the greatest benefit (if any) or the greatest harm.

A challenging question remains, as to whether younger perimenopausal women experience a different net effect compared with older women studied in randomized trials so far. It is conceivable that time since menopause will affect the inflammatory potential and the responsiveness and density of estrogen receptors, thereby modulating the therapeutic effect of HRT [94]. Support in favor of this hypothesis comes from studies in nonhuman primates: HRT inhibited coronary atherosclerosis progression by $70 \%$ if begun early after ovariectomy in cynomolgus monkeys with little or no atherosclerosis. HRT was ineffective, however, if started two years after ovariectomy (corresponding to six human years; reviewed in [95]). These observations still need to be reproduced in humans. By contrast, data from the Women's Health Initiative suggest similar cardiovascular risk for the age group 50-59 years [8], but the respective analysis using the variable years since menopause instead of age is not yet available.

Finally, the choice of estrogen and gestagen and the mode of application need further definition. Currently, it remains speculative whether combined HRT utilizing
$17 \beta$-estradiol would offer better cardiovascular protection in primary prevention than conjugated equine estrogens. Until now, the large body of evidence in human studies was collected in women using conjugated equine estrogens. This contrasts with the bulk of in vitro and animal experiments, which used $17 \beta$-estradiol preparations. Some encouraging results from studies of $17 \beta$-estradiol on inflammatory markers have been pointed out above. However, the negative results from endpoint trials utilizing combined and unopposed $17 \beta$-estradiol regimens in secondary prevention $[14,15,17]$ have cast doubt on whether this approach conveys a large benefit (i.e. $30-50 \%$ risk reduction) in primary cardiovascular prevention. Currently, no trial addresses this question. Analogously, it is unclear whether selective estrogen receptor modulators [96] will offer a better preventive strategy. This is another expanding field of research, because no concomitant rise in CRP has been reported for these compounds [49,55,97]. Similarly, no clinical evidence yet supports the hypothesis that other routes of estrogen application (transdermal and transnasal), which bypass the liver and avoid an increase in CRP, will result in better clinical outcome.

\section{Conclusions}

Estrogenic compounds, with or without the addition of a gestagen, modulate the inflammatory response in postmenopausal women. Consistent anti-inflammatory effects of HRT compounds are seen with regard to cellular adhesion molecules, but divergent effects are seen on CRP: conjugated equine estrogens consistently increase CRP, whereas $17 \beta$-estradiol seems to elicit a weaker proinflammatory response. Transdermal treatment with $17 \beta$-estradiol does not increase CRP. However, it is unknown whether the rise in CRP induced by oral estrogen, possibly as a consequence of hepatic metabolism, translates into more aggressive inflammation and worse clinical outcome. CRP is the marker for which the best evidence has been collected in both observational and randomized trials. However, this does not necessarily imply that CRP is the most important risk marker/ mediator from a biological perspective compared with upstream cytokines or cell adhesion molecules.

In contrast to observational evidence, data from randomized trials do not support the use of HRT in primary or secondary prevention of CVD and cerebrovascular disease in women aged 60-65 years. Various plausible factors have been identified that might have contributed to this selectively discordant finding. Modulation of inflammation might be one of these factors, or might be an epiphenomenon. It remains an ongoing challenge to identify subgroups of women who will experience the greatest harm or greatest benefit. In this respect, endpoint studies in younger, perimenopausal women are warranted. Information on inflammatory biomarkers in this setting will widen our understanding of atherosclerosis as a modifiable condition.

\section{Acknowledgements}

The authors apologize to colleagues whose work could not be cited because of space constraints. 


\section{References}

1 van der Schouw, Y.T. et al. (1996) Age at menopause as a risk factor for cardiovascular mortality. Lancet 347, 714-718

2 Grady, D. et al. (1992) Hormone therapy to prevent disease and prolong life in postmenopausal women. Ann. Intern. Med. 117, 1016-1037

3 Grodstein, F. et al. (2001) Postmenopausal hormone use and secondary prevention of coronary events in the nurses' health study. A prospective, observational study. Ann. Intern. Med. 135, 1-8

4 Grodstein, F. et al. (1996) Postmenopausal estrogen and progestin use and the risk of cardiovascular disease. N. Engl. J. Med. 335, 453-461

5 Stampfer, M.J. and Colditz, G.A. (1991) Estrogen replacement therapy and coronary heart disease: a quantitative assessment of the epidemiologic evidence. Prev. Med. 20, 47-63

6 Angerer, P. et al. (2001) The effect of oral postmenopausal hormone replacement therapy on atherosclerosis in carotid arteries - a randomized, controlled trial. Arterioscler. Thromb. Vasc. Biol. 21, 262-268

7 Hodis, H.N. et al. (2001) Estrogen in the prevention of atherosclerosis. A randomized, double-blind, placebo-controlled trial. Arch. Intern. Med. 135, 939-953

8 Manson, J.E. et al. (2003) Estrogen plus progestin and the risk of coronary heart disease. N. Engl. J. Med. 349, 523-534

9 Hulley, S. et al. (1998) Randomized trial of estrogen plus progestin for secondary prevention of coronary heart disease in postmenopausa women. Heart and estrogen/progestin replacement study (HERS) research group. J.A.M.A. 280, 805-813

10 Grady, D. et al. (2002) Cardiovascular disease outcomes during 6.8 years of hormone therapy: heart and estrogen/progestin replacement study follow-up (HERS II). J.A.M.A. 288, 49-57

11 Herrington, D.M. et al. (2000) Effects of estrogen replacement on the progression of coronary artery atherosclerosis. N. Engl. J. Med. 343 $522-529$

12 Hsia, J. et al. (2000) Peripheral arterial disease in randomized trial of estrogen with progestin in women with coronary heart disease: the heart and estrogen/progestin replacement study. Circulation 102 2228-2232

13 Simon, J.A. et al. (2001) Postmenopausal hormone therapy and risk of stroke: the heart and estrogen-progestin replacement study (HERS). Circulation 103, 638-642

14 Viscoli, C.M. et al. (2001) A clinical trial of estrogen-replacement therapy after ischemic stroke. N. Engl. J. Med. 345, 1243-1249

15 Clarke, S.C. et al. (2002) A study of hormone replacement therapy in postmenopausal women with ischaemic heart disease: the Papworth HRT atherosclerosis study. B.J.O.G. 109, 1056-1062

16 Cherry, N. et al. (2002) Oestrogen therapy for prevention of reinfarction in postmenopausal women: a randomised placebo controlled trial. Lancet 360, 2001-2008

17 Waters, D.D. et al. (2002) Effects of hormone replacement therapy and antioxidant vitamin supplements on coronary atherosclerosis in postmenopausal women: a randomized controlled trial. J.A.M.A $288,2432-2440$

18 Hodis, H.N. et al. (2003) Hormone therapy and the progression of coronary-artery atherosclerosis in postmenopausal women. N. Engl. J. Med. 349, 535-545

19 Grady, D. et al. (2000) Postmenopausal hormone therapy increases risk for venous thromboembolic disease. The Heart and Estrogen/ progestin Replacement Study. Ann. Intern. Med. 132, 689-696

20 US Preventative Services Task Force, (2002) Postmenopausal hormone replacement therapy for primary prevention of chronic conditions: recommendations and rationale. Ann. Intern. Med. 137, 834-839

21 Grodstein, F. et al. (2003) Understanding the divergent data on postmenopausal hormone therapy. N. Engl. J. Med. 348, 645-650

22 Cid, M.C. et al. (1994) Estradiol enhances leukocyte binding to tumor necrosis factor (TNF)-stimulated endothelial cells via an increase in TNF-induced adhesion molecules E-selectin, intercellular adhesion molecule type 1 , and vascular cell adhesion molecule type 1. J. Clin. Invest. 93, 17-25

23 Morales, D.E. et al. (1995) Estrogen promotes angiogenic activity in human umbilical vein endothelial cells in vitro and in a murine model. Circulation 91, 755-763

24 Herrington, D.M. (1999) The HERS trial results: paradigms lost? Ann. Intern. Med. 131, 463-466
25 Ross, R. (1999) Atherosclerosis - an inflammatory disease. N. Engl. J. Med. 340, 115-126

26 Libby, P. et al. (2002) Inflammation and atherosclerosis. Circulation 105, 1135-1143

27 Ridker, P.M. et al. (1998) Prospective study of C-reactive protein and the risk of future cardiovascular events among apparently healthy women. Circulation 98, 731-733

28 Ridker, P.M. et al. (1999) Hormone replacement therapy and increased plasma concentration of C-reactive protein. Circulation 100, 713-716

29 Libby, P. (2000) Changing concepts of atherogenesis. J. Intern. Med. 247, 349-358

30 Ridker, P.M. et al. (2000) C-reactive protein and other markers of inflammation in the prediction of cardiovascular disease in women N. Engl. J. Med. 342, 836-843

31 Yeh, E.T. et al. (2001) C-reactive protein: linking inflammation to cardiovascular complications. Circulation 104, 974-975

32 Pradhan, A.D. et al. (2002) Inflammatory biomarkers, hormone replacement therapy, and incident coronary heart disease - prospective analysis from the women's health initiative observational study. J.A.M.A. 288, 980-987

33 Weinhold, B. and Ruther, U. (1997) Interleukin-6-dependent and -independent regulation of the human C-reactive protein gene. Biochem. J. 327, 425-429

34 Libby, P. and Ridker, P.M. (1999) Novel inflammatory markers of coronary risk - theory versus practice. Circulation 100, 1148-1150

35 Pearson, T.A. et al. (2003) Markers of inflammation and cardiovascular disease. Application to clinical and public health practice. A statement for healthcare professionals from the Centers for Disease Control and Prevention and the American Heart Association. Circulation 107, 499-511

36 Mulvihill, N.T. et al. (2001) Relationship between intracoronary and peripheral expression of soluble cell adhesion molecules. Int. J. Cardiol. 77, 223-229

37 Nilsson, S. et al. (2001) Mechanisms of estrogen action. Physiol. Rev. 81, 1535-1565

38 Gruber, C.J. et al. (2002) Production and actions of estrogens. N. Engl. J. Med. 346, 340-352

39 Losordo, D.W. and Isner, J.M. (2001) Estrogen and angiogenesis. Arterioscler. Thromb. Vasc. Biol. 21, 6-12

40 Gimbrone, M.A. (1995) Vascular endothelium: an integrator of pathophysiologic stimuli in atherosclerosis. Am. J. Cardiol. 75, $67 \mathrm{~B}-70 \mathrm{~B}$

41 Koh, K.K. et al. (2001) Vascular effects of synthetic or natural progestagen combined with conjugated equine estrogen in healthy postmenopausal women. Circulation 103, 1961-1966

42 Koh, K.K. et al. (2001) Effect of hormone replacement therapy on nitric oxide bioactivity and monocyte chemoattractant protein-1 levels. Int. J. Cardiol. 81, 43-50

43 Störk, S. et al. (2002) The effect of $17 \beta$-estradiol on endothelial and inflammatory markers in postmenopausal women - a randomized controlled trial. Atherosclerosis 165, 301-307

44 Störk, S. et al. (2002) The effect of $17 \beta$-estradiol on MCP-1 serum levels in postmenopausal women. Cardiovasc. Res. 53, 642-649

45 Cushman, M. et al. (1999) Effect of postmenopausal hormones on inflammation-sensitive proteins: the postmenopausal estrogen/ progestin interventions (PEPI) study. Circulation 100, 717-722

46 Koh, K.K. et al. (1999) Vascular effects of estrogen and vitamin E therapies in postmenopausal women. Circulation 100, 1851-1857

47 Koh, K.K. et al. (1999) Vascular effects of estrogen and cholesterollowering therapies in hypercholesterolemic postmenopausal women. Circulation 99, 354-360

48 Scarabin, P.Y. et al. (1999) Hormone replacement therapy and circulating ICAM-1 in postmenopausal women - a randomised controlled trial. Thromb. Haemost. 81, 673-675

49 Blum, A. et al. (2000) Effects of estrogen and the selective estrogen receptor modulator raloxifene on markers of inflammation in postmenopausal women. Am. J. Cardiol. 86, 892-895

50 Sbarouni, E. et al. (2000) Cell adhesion molecules in relation to simvastatin and hormone replacement therapy in coronary artery disease. Eur. Heart J. 21, 975-980

51 Zanger, D. et al. (2000) Divergent effects of hormone therapy on serum markers of inflammation in postmenopausal women with coronary 
artery disease on appropriate medical management. J. Am. Coll. Cardiol. 36, 1797-1802

52 Seljeflot, I. et al. (2000) Reduced expression of endothelial cell markers after long-term transdermal hormone replacement therapy in women with coronary artery disease. Thromb. Haemost. 83, 944-948

53 Goudev, A. et al. (2002) Effects of low dose hormone replacement therapy on markers of inflammation in postmenopausal women. Maturitas 43, 49-53

54 Koh, K.K. et al. (2002) Statin attenuates increase in C-reactive protein during estrogen replacement therapy in postmenopausal women. Circulation 105, 1531-1533

55 Walsh, B.W. et al. (2000) The effects of hormone replacement therapy and raloxifene on C-reactive protein and homocysteine in healthy postmenopausal women: a randomized, controlled trial. J. Clin. Endocrinol. Metab. 85, 214-218

56 Koh, K.K. et al. (2002) Effects of continuous combined hormone replacement therapy on inflammation in hypertensive and/or overweight postmenopausal women. Arterioscler. Thromb. Vasc. Biol. 22, 1459-1464

57 Sites, C.K. et al. (2002) Menopause-related differences in inflammation markers and their relationship to body fat distribution and insulinstimulated glucose disposal. Fertil. Steril. 77, 128-135

58 Sumino, H. et al. (2000) Serum level of vascular endothelial growth factor is decreased by hormone replacement therapy in postmenopausal women without hypercholesterolemia. Atherosclerosis 148, 189-195

59 Ylikorkala, O. et al. (1995) Postmenopausal hormonal replacement decreases plasma levels of endothelin-1. J. Clin. Endocrinol. Metab. 80, 3384-3387

60 Wilcox, J.G. et al. (1997) Endothelin levels decrease after oral and nonoral estrogen in postmenopausal women with increased cardiovascular risk factors. Fertil. Steril. 67, 273-277

61 Best, P.J. et al. (1998) The effect of estrogen replacement therapy on plasma nitric oxide and endothelin-1 levels in postmenopausal women. Ann. Intern. Med. 128, 285-288

62 Chen, F.P. et al. (1998) Effects of hormone replacement therapy on cardiovascular risk factors in postmenopausal women. Fertil. Steril. $69,267-273$

63 Ylikorkala, O. et al. (1998) The long-term effects of oral and transdermal postmenopausal hormone replacement therapy on nitric oxide, endothelin-1, prostacyclin, and thromboxane. Fertil. Steril. 69, 883-888

64 van Baal, W.M. et al. (1999) Long-term effects of combined hormone replacement therapy on markers of endothelial function and inflammatory activity in healthy postmenopausal women. Fertil. Steril. 71, 663-670

65 Anwaar, I. et al. (2000) Hormone replacement therapy in healthy postmenopausal women. Effects on intraplatelet cyclic guanosine monophosphate, plasma endothelin-1 and neopterin. J. Intern. Med. $247,463-470$

66 Saltevo, J. et al. (2000) Plasma endothelin in postmenopausal women with type 2 diabetes mellitus and metabolic syndrome: a comparison of oral combined and transdermal oestrogen-only replacement therapy. Diabetes Obes. Metab. 2, 293-298

67 Webb, C.M. et al. (2000) 17ß-Estradiol decreases endothelin-1 levels in the coronary circulation of postmenopausal women with coronary artery disease. Circulation 102, 1617-1622

68 Simoncini, T. et al. (2000) Interaction of oestrogen receptor with the regulatory subunit phophatidylintositol-3OH kinase. Nature 407, $538-541$

69 Saitta, A. et al. (2001) Randomized, double-blind, placebo-controlled study on effects of raloxifene and hormone replacement therapy on plasma NO concentrations, endothelin-1 levels, and endotheliumdependent vasodilation in postmenopausal women. Arterioscler. Thromb. Vasc. Biol. 21, 1512-1519

70 Cid, M.C. et al. (2002) Estrogens and the vascular endothelium. Ann. N.Y. Acad. Sci. 966, 143-157

$71 \mathrm{Koh}, \mathrm{K} . \mathrm{K}$. et al. (1997) Effects of hormone therapy on inflammatory cell adhesion molecules in postmenopausal healthy women. Am. J. Cardiol. 80, 1505-1507

72 Cushman, M. et al. (1999) Hormone replacement therapy, inflammation, and hemostasis in elderly women. Arterioscler. Thromb. Vasc. Biol. 19, 893-899
73 de Valk-de Roo, G.W. et al. (1999) Both raloxifene and estrogen reduce major cardiovascular risk factors in healthy postmenopausal women: A 2-year, placebo-controlled study. Arterioscler. Thromb. Vasc. Biol. 19, $2993-3000$

74 Luyer, M.D.P. et al. (2001) Prospective randomized study of effects of unopposed estrogen replacement therapy on markers of coagulation and inflammation in postmenopausal women. J. Clin. Endocrinol. Metab. 86, 3629-3634

75 Lamon-Fava, S. et al. (2003) Effect of hormonal replacement therapy on C-reactive protein and cell-adhesion molecules in postmenopausal women. Am. J. Cardiol. 91, 252-254

76 Wakatsuki, A. et al. (2002) Effect of medroxyprogesterone acetate on vascular inflammatory markers in postmenopausal women receiving estrogen. Circulation 105, 1436-1439

77 van Baal, W.M. et al. (1999) Increased C-reactive protein levels during short-term hormone replacement therapy in healthy postmenopausal women. Thromb. Haemost. 81, 925-928

78 Sattar, N. et al. (1999) Hormone replacement therapy and sensitive C-reactive protein concentrations in women with type-2 diabetes. Lancet $354,487-488$

79 Decensi, A. et al. (2002) Effect of transdermal estradiol and oral conjugated estrogen on C-reactive protein in retinoid-placebo trial in healthy women. Circulation 106, 1224-1228

80 Post, M.S. et al. (2002) Effects of transdermal and oral oestrogen replacement therapy on C-reactive protein levels in postmenopausal women: a randomised, placebo-controlled trial. Thromb. Haemost. 88, $605-610$

81 Lowe, G.D. et al. (2001) Different effects of oral and transdermal hormone replacement therapies on factor IX, APC resistance, t-PA, PAI and C-reactive protein - a cross-sectional population survey. Thromb. Haemost. 86, 550-556

82 Vehkavaara, S. et al. (2001) Effects of oral and transdermal estrogen replacement therapy on markers of coagulation, fibrinolysis, inflammation and serum lipids and lipoproteins in postmenopausal women. Thromb. Haemost. 85, 619-625

83 Modena, M.G. et al. (2002) Effects of hormone replacement therapy on C-reactive protein levels in healthy postmenopausal women: comparison between oral and transdermal administration of estrogen. Am. J. Med. 113, 331-334

84 Rifai, N. and Ridker, P.M. (2001) High-sensitivity C-reactive protein: a novel and promising marker of coronary heart disease. Clin. Chem. 47, 403-411

85 Harris, T.B. et al. (1999) Associations of elevated interleukin-6 and C-reactive protein levels with mortality in the elderly. Am. J. Med. 106, 506-512

86 Danesh, J. et al. (2000) Low grade inflammation and coronary heart disease: prospective study and updated meta-analyses. BMJ 321, 199-204

87 Ridker, P.M. et al. (2001) Soluble P-selectin and the risk of future cardiovascular events. Circulation 103, 491-495

88 Ridker, P.M. et al. (1998) Inflammation, pravastatin, and the risk of coronary events after myocardial infarction in patients with average cholesterol levels. Circulation 98, 839-844

89 Albert, M.A. et al. (2001) Effect of statin therapy on C-reactive protein levels. The pravastatin inflammation/CRP evaluation (PRINCE): a randomized trial and cohort study. J.A.M.A. 286, 64-70

90 Jialal, I. et al. (2001) Effect of hydroxymethyl glutaryl coenzyme A reductase therapy on high sensitive C-reactive protein levels. Circulation 103, 1933-1935

91 Bots, M.L et al. The osteoporosis prevention and arterial effects of tribolone (OPAL) study: design and baseline characteristics Control. Clin. Trial. (in press)

92 McDonough, P.G. (2002) The randomized world is not without its imperfections: reflections on the Women's Health Initiative Study. Fertil. Steril. 78, 951-956

93 Beral, V. et al. (2003) Breast cancer and hormone replacement therapy in the million women study. Lancet $362,419-427$

94 Karas, R.H. and Clarkson, T.B. (2003) Considerations in interpreting the cardiovascular effects of hormone replacement therapy observed in the WHI: timing is everything. Menopausal Med. 10, 8-11

95 Mikkola, T.S. and Clarkson, T.B. (2002) Estrogen replacement therapy, atherosclerosis, and vascular function. Cardiovasc. Res. 53, $605-619$ 
96 Barrett-Connor, E. et al. (2002) Raloxifene and cardiovascular events in osteoporotic postmenopausal women: four-year results from the MORE (multiple outcomes of raloxifene evaluation) randomized trial. J.A.M.A. 287, 847-857

97 Cushman, M. et al. (2001) Tamoxifen and cardiac risk factors in healthy women: suggestion of an anti-inflammatory effect. Arterioscler. Thromb. Vasc. Biol. 21, 255-261

98 Caulin-Glaser, T. et al. (1998) Modulation of circulating cellular adhesion molecules in postmenopausal women with coronary artery disease. J. Am. Coll. Cardiol. 31, 1555-1560

99 Imthurn, B. et al. (1997) Differential effects of hormone-replacement therapy on endogenous nitric oxide (nitrite/nitrate) levels in postmenopausal women substituted with $17 \beta$-estradiol valerate and cyproterone acetate or medroxyprogesterone acetate. J. Clin. Endocrinol. Metab. 82, 388-394

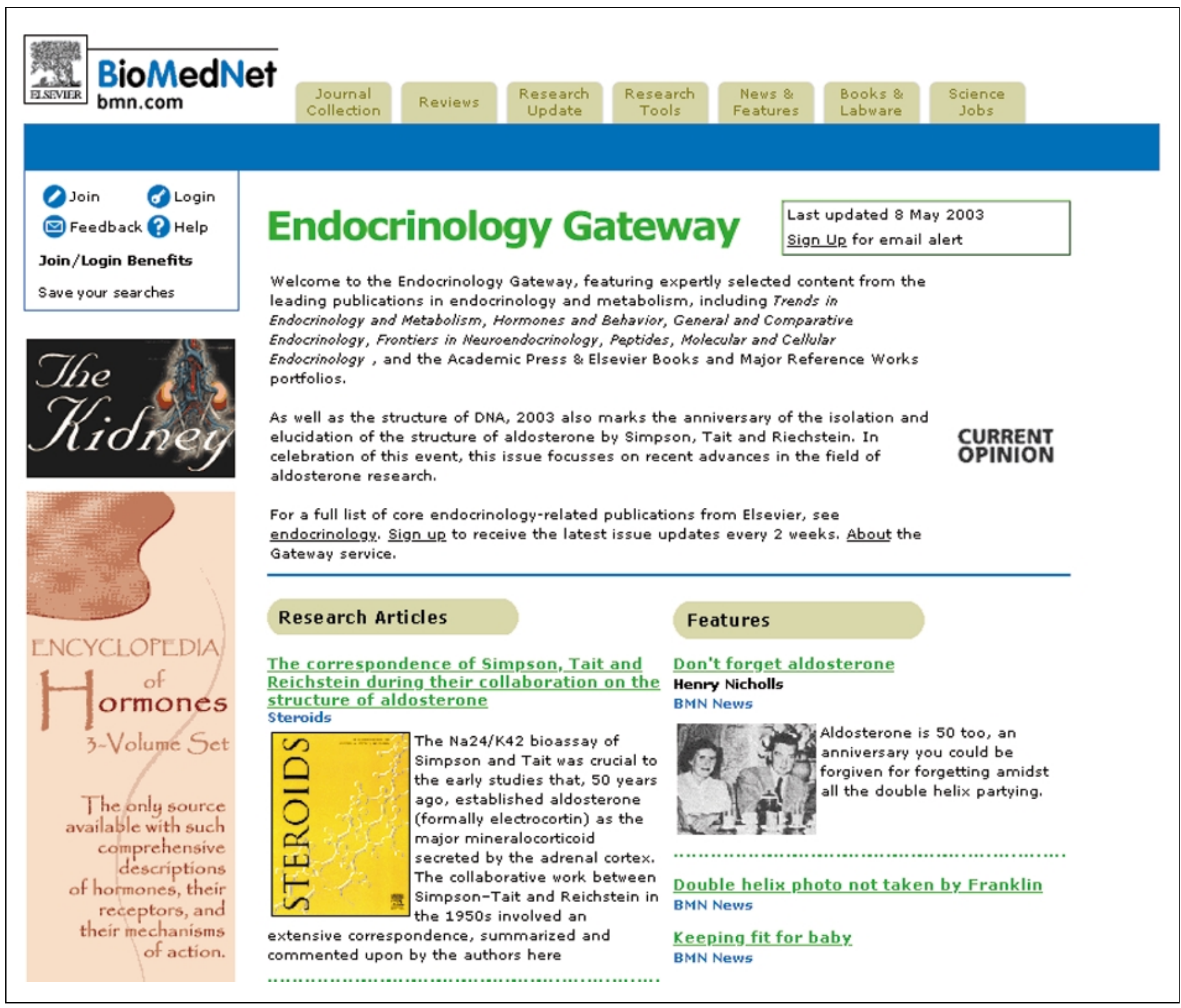

